\title{
Laboratory-Based Deployment and Investigation of PMU and OpenPDC Capabilities
}

\author{
Mohammad Golshani (mohammad.Golshani@brunel.ac.uk), Gareth A. Taylor (gareth.taylor@brunel.ac.uk) \\ Ioana Pisica (oana.pisica@brunel.ac.uk), Phillip Ashton (phillip.ashton@brunel.ac.uk)
}

Brunel Institute of Power Systems, Brunel University, Uxbridge, Middlesex, UB8 3PH, UK

Keywords: OpenPDC, PMU, RTU, SCADA, WAMS

\begin{abstract}
Power systems operate in a more complicated condition than they used to. Changes in the wholesale electricity market alongside the difficulties in upgrading the transmission system have caused transmission systems to face more challenging network wide issues. Therefore, having a Wide Area Measurement System (WAMS) is vital with regard to ensuring secure and reliable operation. Traditionally, Supervisory Control and Data Acquisition (SCADA) systems were designed for monitoring of the power systems by polling the Remote Terminal Units (RTUs) at all the substations. However, the gathering of data is done at a slow rate of every few seconds so the monitoring is relatively static and infrequent. By using Phasor Measurement Units (PMUs) data can be provided in higher rates, commonly once every cycle, and with higher accuracy. The measured data from PMU is transmitted to a central location called Phasor Data Concentrator (PDC), where data can be collected, managed and processed. PMU-based WAMS has a significant role to play in enabling "Smart Grids" at the transmission level. Brunel University has deployed a PMU and local PDC using openPDC to visualize, process and archive grid measured data locally in the laboratory. In this paper we present an investigation on openPDC features and a preliminary study that has been performed based on the PMU deployment.
\end{abstract}

\section{Introduction}

Operation of power systems has become more complicated and therefore encounters more challenges. From the generation viewpoint, power industries have been deregulated and more independent power generators contribute as suppliers. In addition, there is a wider penetration of renewable and variable sources of generation. On the other hand, by the presence of less predictable load patterns and more power electronics driven sensitive loads, there is a higher requirements in providing reliability and power quality. Meanwhile, difficulties also exist in upgrading the transmission system proportional to the growing generations and loads. These are some illustrations of why Wide Area Measurement System (WAMS) must be deployed throughout the power system in order to observe, in real-time, the changing state of the electrical power network, take appropriate corrective measures and isolate faults quickly [1, $2]$.

SCADA system has been the essential component for monitoring in power system for years. However it is not effective for the real-time wide area monitoring applications, due to the low data sampling rate and lack of exact time synchronization. The new generation of measurement technology, known as Phasor Measurement Unit (PMU), offers synchronized measurements at higher rate of commonly once every cycle.

A PMU-based WAMS is a system in which PMUs measure power system parameters including frequency, voltage and current phasors with high accuracy. Meanwhile, the phasors are time-stamped using signals from Global Positioning System (GPS). Afterwards, the time-critical phasor data are collected from various locations in the electrical grid and will be transmitted to a central location called Phasor Data Concentrator (PDC). A PDC receives and time-synchronizes phasor data from geographically distributed PMUs and produces a real-time, time-aligned output data stream. This information will be exploited by Smart Grid applications including visualization tools, state estimation, event optimal control, protection, etc. or can be also stored for future analysis. Figure 1 shows the structure of the WAMS and placement of a PDC [2].

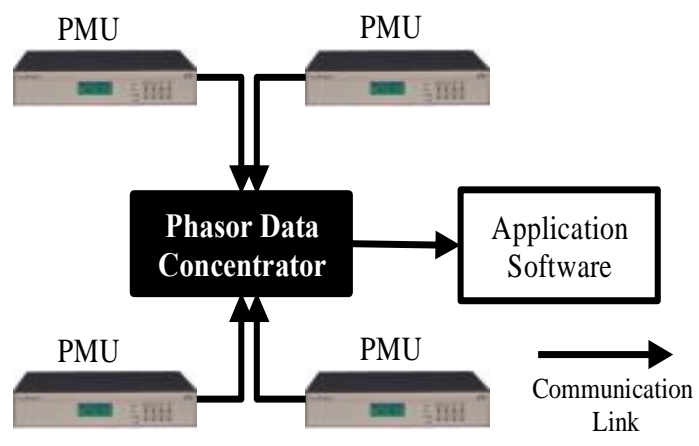

Figure 1: WAMS structure [3]

Therefore, similar to traditional SCADA system, WAMS consists of three layers. The first layer is the section that WAMS interfaces with power system to measure required parameters. This layer is called the Data Acquisition Layer 
and PMUs are located in this section. Layer 2 is where the PMUs measured data are collected and time-aligned. This layer is known as the Data Management Layer and PDC is placed at this layer. Finally, layer 3 is the Application Layer where the sorted PMUs measurements are used by the different kind of functions for the monitoring, control and protection applications [4].

Employing WAMS based on PMU enables dynamic view coverage of the network behaviour and, in turn, improves stability and reliability of the power system operations [5]. In this paper the laboratory-based deployment of phasor measurement technology is investigated and work that has been carried out by Brunel University in this field alongside its future development aims will be presented. The rest of the paper is structured as follows: Section 2 describes the PMUbased monitoring deployment in Brunel University. Section 3 investigates the openPDC and presents its main features. The challenges ahead for the development of WAMS will be discussed in section 4. Hadoop open source framework for cloud computing and common Smart Grid applications for WAMS will be addressed in section 5 and 6 , respectively. Finally, the paper is concluded in section 7 .

\section{PMU-based Monitoring In Brunel University Laboratory}

Employing PMUs in laboratory-based and domestic level simplifies investigation of wide area monitoring system compared to the more complicated case of installing them in substations. There is more equipment required inside a substation for deploying PMUs, such as step-down transformers that bring the three-phase voltage or current level to instrumentation level. Brunel University, London installed a PMU that is connected to the 3 phase $415 \mathrm{~V}$ AC domestic supply level and joined a WAMS originally provided by the SUPERGEN FlexNet Consortium. Currently, PMUs measured synchrophasors from 4 UK Universities are transmitted via the Internet to a server in Ljubljana, Slovenia. As the PMUs are well geographically distributed across the Scottish to England system, as depicted in Figure 2, they provide a good visibility of any oscillatory events through the covered area. The PDC in Slovenia is running WAProtector, which is a system for wide area monitoring, protection and control provided by the ELPROS [6]. The Brunel installed PMU measures and sends 50 samples of parameters per second. The number of measured parameters in PMU output determines the size of data packet. Each data stream consists of measurements such as voltage magnitude, voltage angle, frequency, frequency delta $(\mathrm{dF} / \mathrm{dt})$ etc. It should be mentioned that PMU in Brunel does not measure current. Figure 3 illustrates the measured voltage phasors of the 4 PMUs; the blue one represents the voltage of the Brunel PMU.

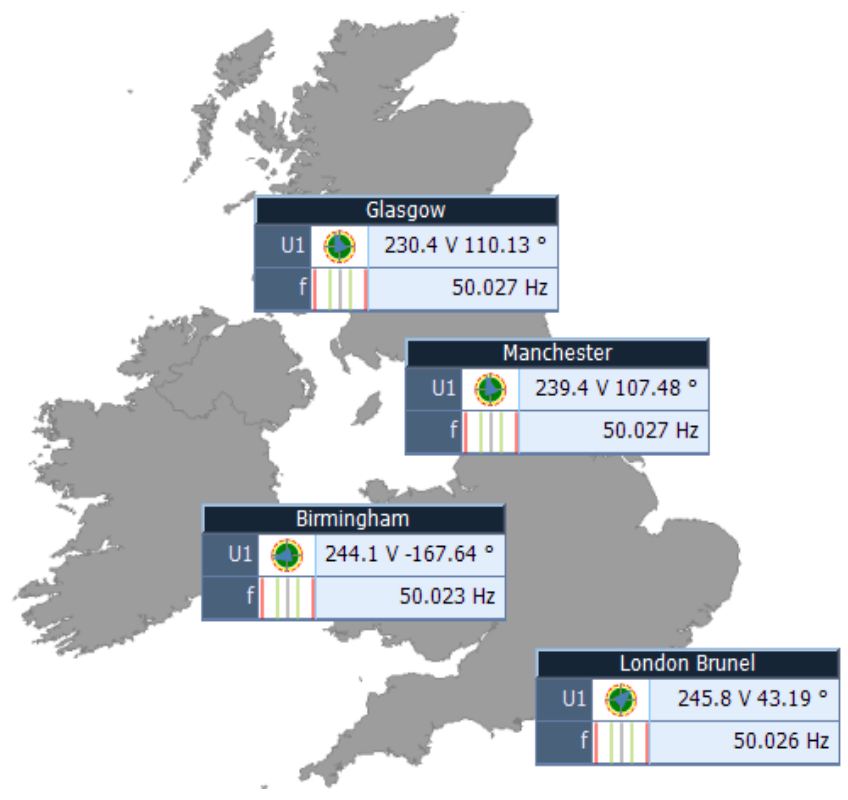

Figure 2: The project PMUs location across the UK

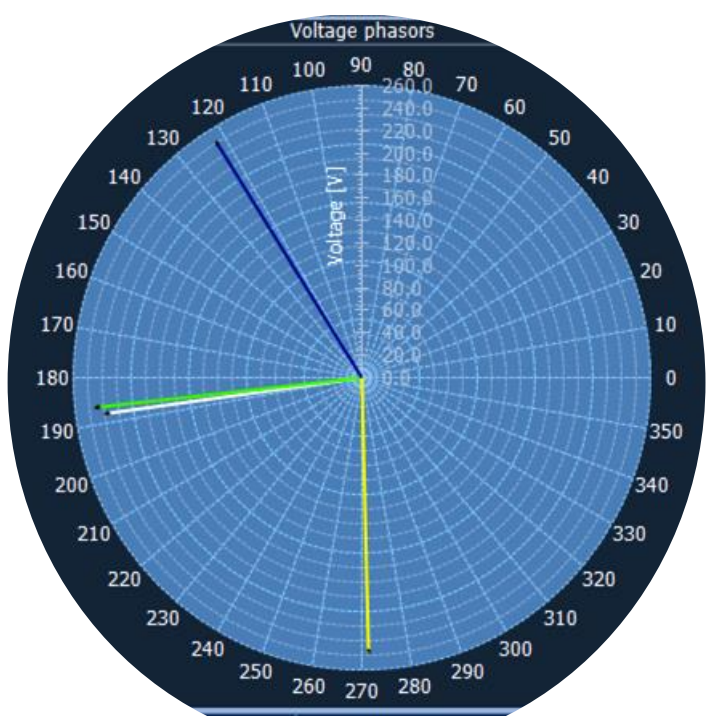

Figure 3: Phasor representation of voltages

However, a local PDC has also been developed recently by using openPDC, which collects measurements locally. The measured data can be visualized online and also forwarded as a continuous data stream to the Historian system, where data will be archived for future analysis. Figures 4 and 5 show a sample of the frequency and voltage variations measured by openPDC for PMU at Brunel over a one-day period. In the next section openPDC and its features will be described. 


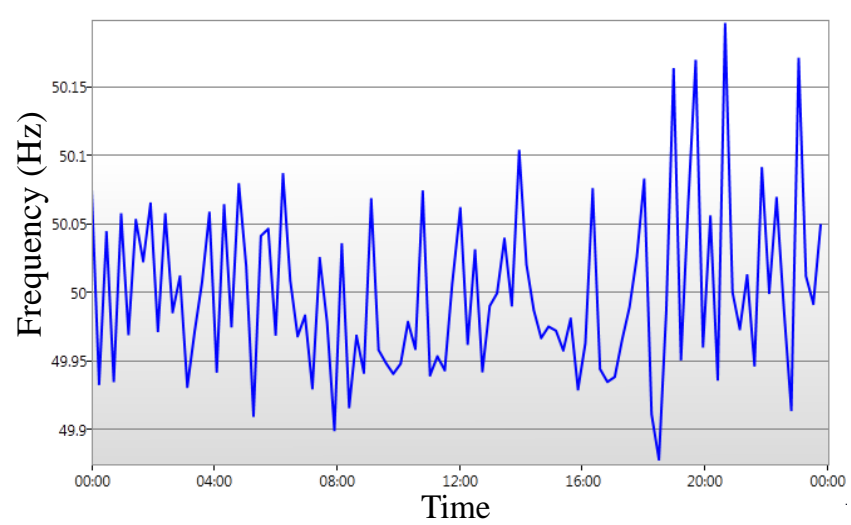

Figure 4: Measured frequency captured from openPDC

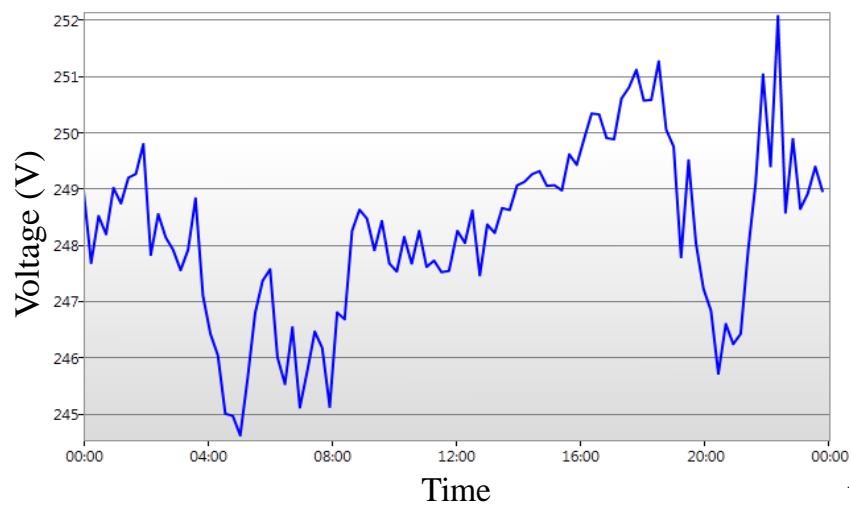

Figure 5: Measured voltage captured from openPDC

\section{Open Source Phasor Data Concentrator}

PDCs have a significant role in WAMS. Earlier PDCs, such as the PBA PDC, were specialized standard alone units and they were not openly available. Whilst openPDC is an open source PDC, which was developed and made available to public by the Tennessee Valley Authority (TVA), in October 2009. It collects, processes and manages data from PMUs or other PDCs. OpenPDC architecturally consists of three layers: the Input, Action and Output Layers. Each layer performs a specific set of functions. Figure 6 shows the adapter based architecture of the openPDC [7, 8].

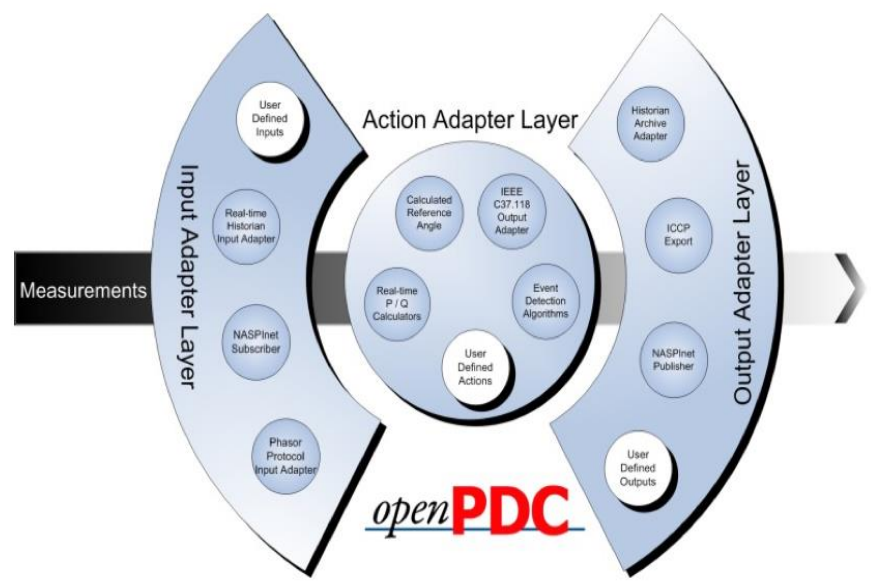

Figure 6: OpenPDC adapter based architecture [8]
The Input Adapter Layer reads streaming data from the measurement devices that may use different protocols. In fact, it allows the parsing of protocol and provides a generic data format. After assigning an ID to the measurement, it will transfer them to the Action Layer. Currently openPDC supports different kinds of protocols including IEEE C37.118-2005 and 2011, IEEE1344, BPA PDCStream, Virginia Tech FNET, Macrodyne, SEL Fast Message, etc. The openPDC deployed in Brunel University currently uses IEEE C37.118 to communicate with the PMU $[9,10]$.

The Action Adapter Layer deals with concentration and processing of the input measurements. One of the important functions of this layer is the phasor time alignment adapter. In this function the measurements received from the Input Layer are sorted by their associated GPS time-stamps and timealigned before transferring them to the next layer. The Action Adapter Layer also provides two more essential functions, which are real-time measurement calculation and real-time event detection. The real-time measurement calculation function uses the measured parameters of the PMUs to calculate new parameters that are not directly collected; For instance, calculation of active and reactive power. It is evident that all the required parameters for the calculation of new parameter must be measured and available by the PMU. As in Brunel University PMU does not measure current, the calculation of new parameters, which are related to the current is not possible. The real-time event detection function monitors the incoming measurements and ensures that they are within the specified limits. Otherwise, it will notify of such an occurrence [9].

Finally, the Output Adapter Layer of the openPDC receives all measured and calculated parameters at the end and queues up data and forward them to either Historian system to archive them for future off-line analysis or to any other defined client systems. The Output Adapter can reencapsulate the data in several protocols. Example of openPDC output types are IEEE C37.118 concentrator output stream, Inter Control Center Protocol (ICCP), Comma Separated Values (CSV) File Export, and Historian Archiving output $[8,9]$.

OpenPDC has various features that make the monitoring of the power systems more convenient. One of them is the ability to replay data stream from a specific time in the past. In this mode, the stored data from the Historian will be retransmitted to the openPDC software and shows the data as if it is coming from the PMU. This near real-time event replay facilitates event analysis and helps the system operator to take appropriate corrective or protective control actions quickly. Another feature of openPDC is that each of the adapter layers functionalities can be improved or extended by user or developer. For example, a new model can be written for Input Adapter to enable support of new protocols. Or a new Action Adapter can be defined to provide particular function, such as calculation of new parameter, detection of specific event, etc. $[10,11]$. 


\section{Challenges and Future Developments}

The main challenges in developing of such a measurement system includes:

There should be sufficient numbers of installed PMUs to test the applications on the openPDC platform. In addition, having more PMUs provides better view of the event propagation in the network. Therefore, more PMUs are required to be employed to send data to openPDC in Brunel University.

In laboratory-based deployment a few PMUs transmit data to the PDC. While for the whole power systems high number of PMUs are installed and they depend on the limited number of PDCs to process and store their data. Therefore, each PDC needs to deal with huge amount of data and the required data storage capacity should be considered. In the case of Brunel University, the PC hard drive works as a server for storing stream of data locally. However, the capacity should be extended for future developments. As more PMUs are deployed in the system, the number of measurements samples increase and the storage requirement is accelerating [9].

Storing and processing a huge volume of PMUs measurements and scanning through terabytes of information to find the particular event will be a big challenge for WAMS. Currently available storage and processing systems, such as Storage Area Network (SAN) and Relational DataBase Management System (RDBMS), have a low read rates and do not work with high resolution time-series data of WAMS. Thus efficient platform is required to interact with huge amount of high-resolution data. Hadoop is the proposed computing and storage framework to be used with openPDC and handle these concerns. In section 5 more information about Hadoop will be provided [12].

Advanced applications that can analyze data and recognize potential problems quickly need to be developed and implemented. These applications provide useful information for operators in order to identify sequence of events and their causes during a power system disturbance. Hence, operators would have more time and better understanding to evaluate the simulation and adopt appropriate control actions [11]. Common Smart Grid applications that exploit these measurements are investigated in the section 6 .

\section{WAMS Applications for Power System}

WAMS brings great potential for improving stability and reliability based on the operational feature of the PMUs. In the literatures, there are a large number of frequently used WAMS applications, however they can be categorized into three main groups of monitoring, protection, and control applications [13].

\section{Power System Monitoring}

State estimation is one of the important parts for monitoring of the power systems. It uses measurements of real and reactive power in line flows and injection point in order to estimate the bus voltage angle and magnitude. Each measurement cycle may take couple of seconds to minutes and during this period system is assumed to be static. But the real system condition is dynamic and oscillation occurs on the flow. By using PMUs the time-stamped measurements can be relayed on a continuous basis and the state vector can follow the dynamics of the system $[14,15]$. PMUs offer a number of possible benefits to the state estimation application including: direct calculation of the state using phasors, faster solution convergence, enhanced observability, improved solution accuracy and robustness, bad data detection and topology error correction, etc [16].

\section{Power System Protection}

Synchronized phasor measurements offer solution to a number of complex protection problems and improve the performance of the protection applications. In general, phasor measurements are particularly effective in enhancing power system protection functions that have a slow response time requirement. Some protection systems that could benefit from PMUs include: adaptive dependability and security, back up protection of distance relays, adaptive out-of-step, angular voltage stability of network, etc [17].

\section{Power system control}

Prior to the emergence of real-time phasor measurements most of the control systems were processed locally due to low time delay. Controllers like variable series capacitor (VSC), universal power flow controllers (UPFCs) and power system stabilizers regulate the grid based on a local feedback. While phasor measurements in addition to remote control of the power systems, could provide direct feedback to these controllers and enable dynamic control of the power systems. Using PMUs to damp the low frequency inter-area oscillations is one of the effective applications of WAMS [15, 17].

\section{Hadoop Framework}

Hadoop is a framework provided by the Apache Foundation as an open source project for running applications on large clusters built of commodity hardware. Whilst openPDC does not offer a cloud computing platform, it utilizes this scalable fault-tolerant distributed system for data storage and processing. Hadoop has two primarily parts: Hadoop Distributed File System (HDFS) and MapReduce programming model $[8,18]$. 
HDFS is the storage system used by Hadoop, Which has master-slave architecture. Accordingly, each cluster consists of a single NameNode and a set of DataNodes. Figure 7 provides an illustration of a high-level architecture for HDFS working principle. When data is transferred to the Hadoop cluster, HDFS creates user0definable number of replication of the data and stores them in blocks on the various DataNodes. NameNode will retain and index of all locations. In this case any node failure will never cause a data loss. Furthermore, this replication of data also enables parallel data reading, which in turn reduces the required time for pulling large data $[10,19]$.

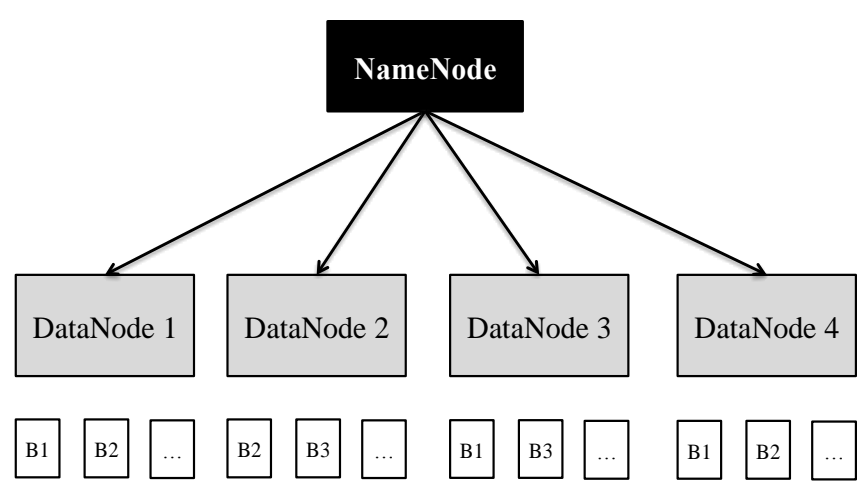

Figure 7: High-level architecture for HDFS working principle [20]

MapReduce is a programming framework designed for parallel processing of huge datasets on distributed sources. This computing model is divided into two parts, Map and Reduce. In the Map step input is divided into smaller segments and distributed to the nodes. Then nodes will process the related segments and produce intermediate outputs separately. In the Reduce step the individual outputs are collected from the nodes and after aggregating they form the main output. Therefore, by running parallel processes, analysis conducted in less time. In fact, Hadoop transfers the processing to the data instead of the conventional procedure of transferring the data to the processing [21].

\section{Conclusion}

In this paper, a preliminary work on the development of WAMS has been provided. PMU-based online monitoring system can significantly improve real-time situation awareness of power system operators. The successful deployment of Smart Grid concept at transmission level will largely depend on the implementation of this technology. OpenPDC released in order to provide open source PDC and its modular design enables processing of time-series measurement data in a protocol independent manner. Its Input, Action and Output Layers deal with measurement acquisition, measurement processing and measurement forwarding, respectively. The openPDC output data is transmitted to either Historian for archiving or to any other applications, which use these measurements for monitoring, protection, or control purposes. Efficiently storing, processing and analyzing a huge volume of PMUs measurements are some of the challenges in developing of such a system. In this regard, Hadoop provides a scalable fault-tolerant distributed system for data storage and processing. It splits data into chunks and distributed them across the nodes in cluster and also enables parallel processing.

\section{References}

[1] Ballance, J.W., Bhargava, B., Rodriguez, G.D., "Monitoring Power System Dynamics using Phasor Measurement Technology for Power System Dynamic Security Assessment," in Proc. IEEE Bologna Power Tech Conference, Vol. 3, June 2003, pp. 1-7.

[2] Gurbiel, M., Komarnicki, P., Styczynski, Z.A., Kereit, M., Blumschein, J., Buchholz, B.M., "Usage of Phasor Measurement Units for Industrial Applications," in Proc. Power and Energy Society General Meeting, July 2011, pp. 1-5.

[3] Elias Karam, "Implementation and Simulation of Communication Network for Wide Area Monitoring and control Systems in OPNET," Master of Science Thesis, KTH, Stockholm, Sweden, 2008.

[4] Chenine, M., Nordstrom, L., "Modeling and Simulation of Wide-Area Communication for Centralized PMUBased Applications," IEEE Power Delivery, vol.26, no.3, pp.1372-1380, July 2011.

[5] Lixia, M., Muscas, C., Sulis, S., "On the accuracy specifications of Phasor Measurement Units," in Proc. Instrumentation and Measurement Technology Conference, May 2010, pp. 1435-1440.

[6] P. M. Ashton, G. A. Taylor, M. R. Irving, A. M. Carter and M. E. Bradley, "Prospective Wide Area Monitoring of the Great Britain Transmission System using Phasor Measurement Units," in Proc. IEEE Power Engineering Society General Meeting Conf. July 2012.

[7] L. Vanfretti and J.H. Chow, "Synchrophasor Data Applications for Wide-Area Systems," In Proceedings of the Power Systems Computation Conference 2011, Stockholm, Sweden.

[8] "OpenPDC: The Open Source Phasor Data Concentrator," online: http://openpdc.codeplex.com/.

[9] Chenine, M.; Vanfretti, L.; Bengtsson, S.; Nordstroum, L., "Implementation of an experimental wide-area monitoring platform for development of synchronized phasor measurement applications," Power and Energy Society General Meeting, 2011 IEEE, pp.1-8, 24-29 July 2011.

[10] Trachian, P., "Machine learning and windowed subsecond event detection on PMU data via Hadoop and the openPDC," Power and Energy Society General Meeting, 2010 IEEE, pp.1-5, 25-29 July 2010.

[11] Guorui Zhang; Kai Sun; Chen, H.; Carroll, R.; Liu, Y., "Application of synchrophasor measurements for improving operator situational awareness," Power and Energy Society General Meeting, 2011 IEEE, pp.1-8, 2429 July 2011. 
[12] "Hadoop as the Platform for the Smartgrid at TVA," Cloudera, 26 Aug, 2010, online:

http://www.cloudera.com/

[13] A.G. Phadke, J.S. Thorp, "Communication needs for wide area measurement applications," Critical Infrastructure (CRIS), 5th International Conference, September 2010.

[14] Di Cao Andonovic, I., "Research on backbone communication network in smart grid by using OPNET," 2011 IEEE International Conference on Smart Measurements for Future Grids (SMFG), pp.130-134, 14-16 Nov. 2011.

[15] Naduvathuparambil, B., Valenti, M.C., Feliachi, A., "Communication delays in wide area measurement systems," Proceedings of the Thirty-Fourth Southeastern Symposium on System Theory, pp. 118- 122, 2002.

[16] Tholomier, D., Kang, H., Cvorovic, B., "Phasor measurement units: Functionality and applications," Power Systems Conference, pp.1-12, 10-13 March 2009.

[17] De La Ree, J., Centeno, V., Thorp, J.S., Phadke, A.G., "Synchronized Phasor Measurement Applications in Power Systems," IEEE Transactions on Smart Grid, vol.1, no.1, pp.20-27, June 2010.

[18] Da-Wei Zhang, Fu-Quan Sun, Xu Cheng, Chao Liu, "Research on hadoop-based enterprise file cloud storage system," 3rd International Conference on Awareness Science and Technology (iCAST), pp.434-437, 27-30 Sept. 2011.

[19] "HDFS Architecture," online: http://hadoop.apache.org

[20] Ramesh Natarajan, "Apache Hadoop Fundamentals," online: http://www.thegeekstuff.com/

[21] Songqing Duan, Bin Wu, Bai Wang, Juan Yang, "Design and implementation of parallel statiatical algorithm based on Hadoop's MapReduce model," IEEE International Conference on Cloud Computing and Intelligence Systems (CCIS), pp.134-138, 15-17 Sept. 2011. 